\title{
A novel KRT5 mutation associated with generalized severe epidermolysis bullosa simplex in a 2-year-old Chinese boy
}

\author{
JIA ZHANG ${ }^{*}$, MING YAN* ${ }^{*}$, JIANYING LIANG, MING LI and ZHIRONG YAO \\ Department of Dermatology, Xinhua Hospital Affiliated to Shanghai Jiaotong University School of Medicine, \\ Shanghai 200092, P.R. China
}

Received April 14, 2015; Accepted May 11, 2016

DOI: $10.3892 /$ etm.2016.3717

\begin{abstract}
Mutations in keratin 5 (KRT5) or KRT14 genes are responsible for the most severe form of epidermolysis bullosa simplex (EBS), which is EBS generalized severe (EBS-gen sev). To date, only four pathogenic mutations (p.Arg165Ser and p.Lys199Asn in KRT5; p.Arg125Cys and p.Arg125His in KRT14) have been reported to be responsible for EBS-gen sev in the Chinese population. In the present study, a 2-year-old Chinese boy was clinically suspected to suffer from EBS, and thus Sanger sequencing was performed in the extracted genomic DNA samples from the patient, his parents and 100 healthy controls. A novel de novo heterozygous missense mutation c.503A $>$ G (p.Glu168Gly) located at the $\mathrm{N}$-terminal end segment of the 1A domain in KRT5 was identified by molecular analysis. In silico analysis tools were used to predict the pathogenicity of the novel missense mutation. A diagnosis of EBS-gen sev was thus confirmed according to the clinical presentations and molecular results.
\end{abstract}

\section{Introduction}

Epidermolysis bullosa simplex (EBS) refers to a number of inherited disorders characterized by mechanical stress-induced blistering of the skin (1). EBS comprises three primary types: Localized [EBS-loc; Online Mendelian Inheritance in Man (OMIM) no. 131800], generalized severe (EBS-gen sev; OMIM no. 131760) and generalized intermediate (EBS-gen intermed; OMIM no. 131900) (1). The ultrastructural pathogenesis of EBS-gen sev is the collapse of keratin filaments

Correspondence to: Professor Zhirong Yao or Dr Ming Li, Department of Dermatology, Xinhua Hospital, Shanghai Jiaotong University School of Medicine, 1665 Kongjiang Road, Shanghai 200092, P.R. China

E-mail: zryaosmu@sohu.com

E-mail: aypyslm@163.com

\section{*Contributed equally}

Key words: epidermolysis bullosa simplex, mutation analysis, keratin 5 in basal epidermal cells, resulting in basal cell cytolysis and sequent intraepidermal blister formation, which can differ from other subtypes (2-4). EBS-gen sev typically presents with characteristic features of large, generalized blisters in early infants, and small, clustered (herpetiform) blisters in childhood. In neonates and infants, EBS-gen sev is life-threatening as cutaneous lesions are typically severe, resulting in difficulties in feeding and care (5-7). Subsequent to infancy, particularly during late childhood and adulthood, the prognosis is favorable (5-7). Mutations located at the highly conserved $\alpha$-helical end segments of the 1A domain of keratin 5 (KRT5) and 2B domain of KRT14, also known as helix initiation peptide (HIP) and helix termination peptide (HTP), respectively, typically result in EBS-gen sev $(8,9)$. To date, four pathogenic mutations have been reported to be responsible for EBS-gen sev in the Chinese population, including p.Arg165Ser and p.Lys199Asn in KRT5, and p.Arg125Cys and p.Arg125His in KRT14 (10-13).

In the present study, molecular genetic tests were performed in a 2-year-old boy with suspected EBS, and a novel missense mutation c.503A $>$ G (p.Glu168Gly) located at the N-terminal end segment of the $1 \mathrm{~A}$ domain of KRT5 (HIP) confirmed a diagnosis of EBS-gen sev.

\section{Materials and methods}

Case. The proband was a 2-year-old boy that was referred to the Department of Dermatology of Xinhua Hospital (Shanghai, China) in October 2013. The patient had been reported to present generalized blisters throughout his body since birth (Fig. 1A-D), which were worsened by friction or trauma and were not treated. No other symptoms were observed other than blisters. At the 9-month follow-up visit a dermatological examination showed that there was no evident improvement (Fig. 1E and F). No other relevant medical history and consanguinity was reported in the patient's family. The patient was suspected to have EBS based on the clinical manifestations, and Sanger sequencing was performed to clarify the diagnosis.

Subjects. The proband, his parents and 100 population-matched healthy controls (the mean age of the healthy controls was 24 years old with a range between 18 to 30 years old and a gender ratio of females/males equal to 1.0) were enrolled in the present study. Subsequent to obtaining written informed consent from the participant's mother, peripheral blood 
A

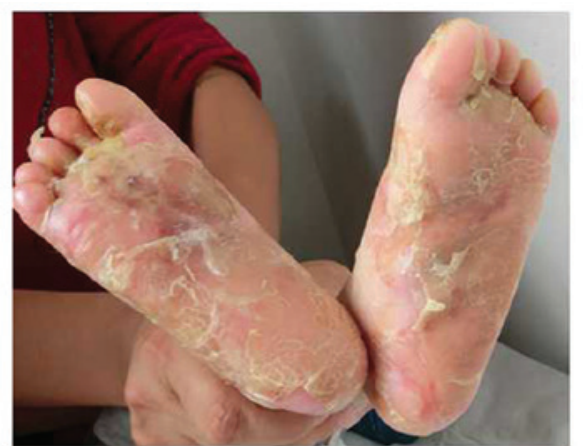

D

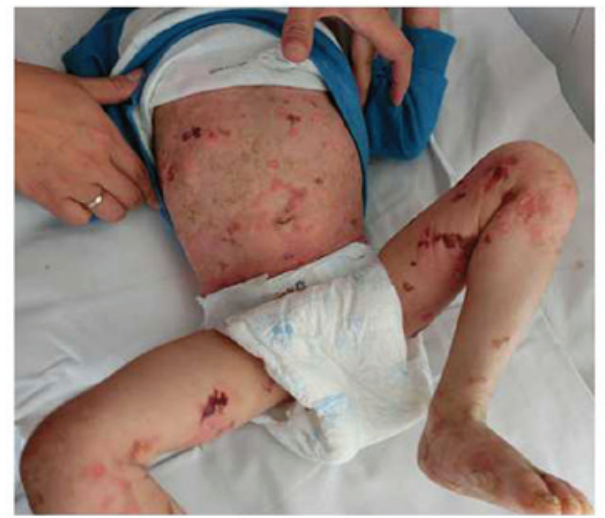

B

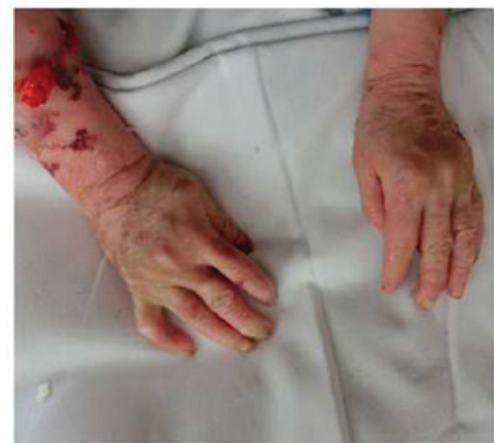

$\mathbf{E}$

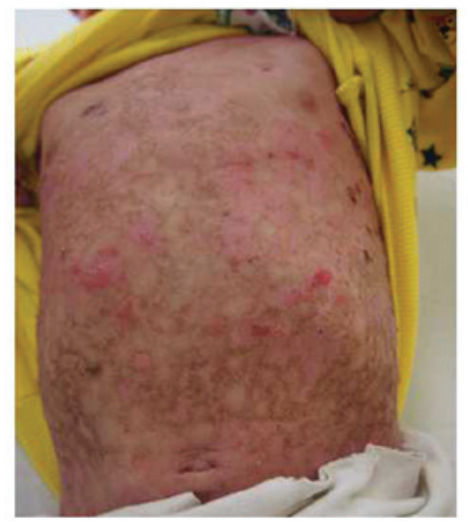

C

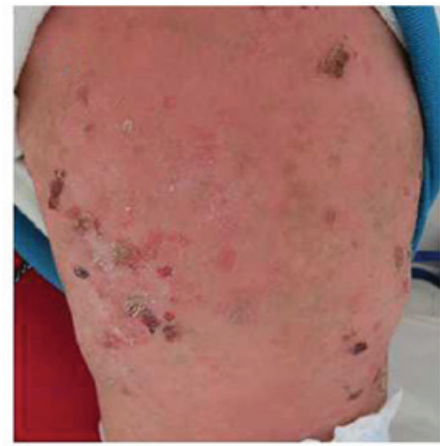

$\mathbf{F}$

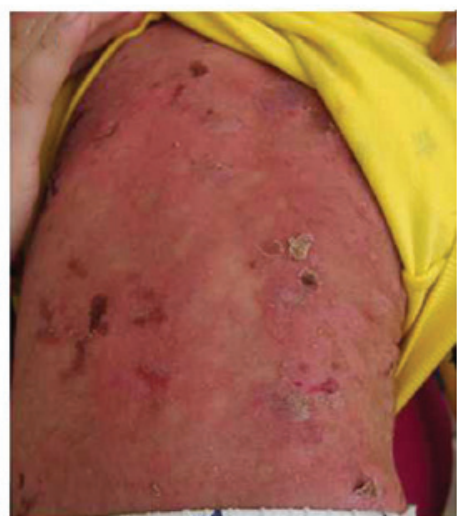

Figure 1. Clinical images of the patient in the current study. (A and B) Dystrophic or absent nails and plantar hyperkeratosis present on the first visit. (C and D) Widespread herpetiform skin blistering with post-inflammatory pigmentation over the entire body. Images A-D were captured when the child was 22 months old. No scarring is present. (E and F) Cutaneous lesions developed after 9 months. Images E and F were captured when the child was 31 months old.

samples were collected for DNA extraction. The present study was approved by the Institutional Review Board of Xinhua Hospital, Shanghai Jiaotong University School of Medicine, and was conducted in accordance with the principles of the Declaration of Helsinki. Ethical approval was obtained from the Ethics Committee of the Xinhua Hospital Affiliated to Shanghai Jiao Tong University School of Medicine.

Methods. Genomic DNA was extracted using a TIANamp Blood DNA kit (Tiangen Biotech Co., Ltd., Beijing, China). Primers flanking all coding exons and intron-exon boundaries of KRT5 and KRT14 were designed using Primer Premier version 5.0 (Premier Biosoft, Palo Alto, CA, USA; Table I). Genomic DNA samples were amplified using polymerase chain reaction (PCR). PCR was performed as follows: A denaturation step at $94^{\circ} \mathrm{C}$ for $5 \mathrm{~min}$; 31 cycles of denaturation at $94^{\circ} \mathrm{C}$ for $30 \mathrm{sec}$, an annealing step for $30 \mathrm{sec}$ (temperature was according to the primers of each fragment), an extension at $72^{\circ} \mathrm{C}$ for $1 \mathrm{~min}$ and an extension at $72^{\circ} \mathrm{C}$ for $1 \mathrm{~min}$. Next, a final extension step was performed at $4^{\circ} \mathrm{C}$ for $5 \mathrm{~min}$, and the experiment was repeated 10-20 times. The PCR products were evaluated by a $2 \%$ agarose gel electrophoresis and were further purified using an AxyPrep DNA Gel Extraction kit (Corning Life Sciences, Corning, NY, USA), according to the manufacturer's instructions. Sanger sequencing was subsequently performed using an ABI PRISM 3730 automated sequencer (Applied Biosystems; Thermo Fisher Scientific, Inc., Waltham, MA, USA). Sequencing results were analyzed by Geneious version 5.6.7 software (Biomatters, Ltd., Auckland, New Zealand). An identified mutation was verified in the corresponding region of the unaffected parents of the proband and 100 population-matched healthy controls. The mutation was described by comparison with the NCBI cDNA reference sequences NM_000424.3 for KRT5 and NM_000526.4 for KRT14.

The potential impact of an amino acid substitution on the structure and function of KRT5 and KRT14 proteins was predicted using the following in silico analysis tools: PolyPhen2 (http://genetics.bwh.harvard. edu/pph2), SIFT (http://sift.bii.a-star.edu.sg) and Mutation Taster (http://www.mutationtaster.org/), which automatically generated the results following input.

\section{Results}

Sequencing results. The results of the present study indicated that mutation sequencing of KRT14 was negative, whereas a novel heterozygous missense mutation, c.503C $>\mathrm{T}$ (p.Glu168Gly), in KRT5 was presented in the patient. This mutation was absent in the patient's unaffected parents and the 100 population-matched healthy controls (Fig. 2).

Functional consequence predictions. Analysis using PolyPhen2 indicated that the mutation c.503A $>$ G (p.Glu168Gly) was 'probably damaging'. Furthermore, SIFT and Mutation Taster software predicted the mutation to be 'deleterious' and 'disease causing', respectively.

\section{Discussion}

The locus of the mutation in KRT5 serves an important role in the phenotype of EBS-gen sev. The majority of causal variants of EBS-gen sev in KRT5 are missense 
Table I. List of the primers of the KRT5 and KRT14 genes.

Primer name

keratin 5-E01_F

keratin 5-E01_R

keratin 5-E02_F

keratin 5-E02_R

keratin 5-E03+4+5_F

keratin 5-E03+4+5_R

keratin 5-E06+7_F

keratin 5-E06+7 R

keratin 5-E08_F

keratin 5-E08_R

keratin 5-E09_F

keratin 5-E09_R

keratin 14-E01_F

keratin 14-E01_R

keratin 14-E02+3_F

keratin 14-E02+3_R

keratin 14-E04-8_F

keratin 14-E04-8_R
Primer Sequence

Annealing temperature $\left({ }^{\circ} \mathrm{C}\right)$

F, forward; R, reverse.

A

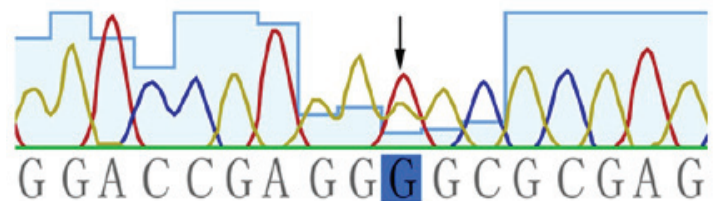

B

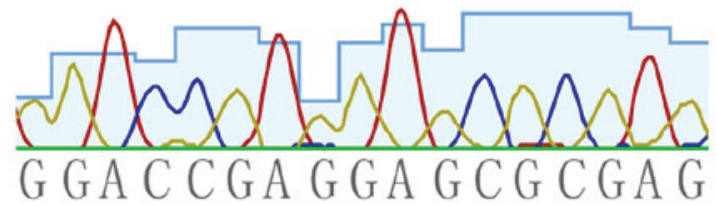

C

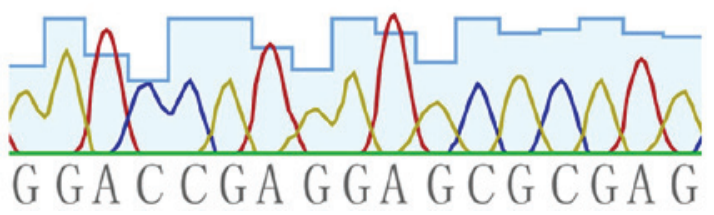

Figure 2. Sequencing results. A novel de novo heterozygous missense mutation c.503A $>$ G (p.Glu168Gly) in the kerain 5 gene was identified by molecular analysis. The arrow indicates the site of mutation. (A) The proband, (B) his father and a (C) normal control.

mutations that exist in the highly conserved regions of HIP and HTP (which are critical for the intermediate filament structure and integrity of the keratin cytoskeleton), and exert a dominant negative effect on the functional protein structure $(2,8,9,14)$. Mutations in other regions are typically associated with the milder subtypes of EBS, namely EBS-loc and EBS-gen intermed $(2,8,14)$.

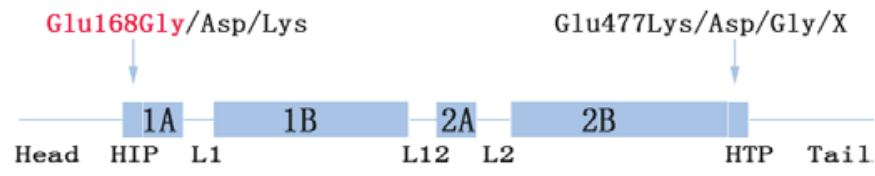

Figure 3. Domain information of the keratin 5 gene and a number of mutations in HIP and HTP responsible for epidermolysis bullosa simplex, generalized severe. The mutation identified in the current study is highlighted red. HIP, helix initation peptide; HTP, helix termination peptide.

The Glu168 site (codon, GAG) is located at the boundary between the head and 1A domain (HIP) (Fig. 3). To date, three relevant substitutions have been reported. More specifically, mutations c.504G $>\mathrm{T}$ (p.Glu168Asp) and c.504G $>\mathrm{C}$ (p.Glu168Asp) result in EBS-gen intermed, while c.502G $>\mathrm{A}$ (p.Glu168Lys) is responsible for EBS-gen sev (15-17). Phenotype severity may be explained by the fact that Glu and Asp are acidic amino acids that share a similar structure and polarity, while Lys and Gly are very different with regards to these features. Contrary to Arg125Cys and Arg125His in the Arg125 site (a CpG-rich hotspot codon) accounting for the majority of mutations in $\operatorname{KRT14}(8,16,18)$, Glu477Lys located at the C-terminal end segments of the helix 2B domain (HTP) was the most common mutation identified in KRT5 (19). This was more common than the corresponding region, Glu168 (Fig. 3; 8,14,16-18).

Dowling-Degos disease (DDD; OMIM no. 179850) or Galli-Galli disease (an acantholytic variant of DDD) are associated with haploinsufficiency of KRT5 caused by heterozygous frameshift/nonsense mutations (such as p.Met1?, p.Gln4* and p.Ile140Asnfs*39) in the head domain of KRT5 (20-22). Furthermore, p.Pro25Leu (in the head domain) and p.Gly550Alafs*77 (in the tail domain) result in a rare 
subtype of EBS, known as EBS with mottled pigmentation (OMIM no. 148040), suggesting that specific regions in KRT5 may be associated with melanin transportation and distribution, and malfunction of which can result in pigmentary phenotypes $(20,23)$.

Although variable phenotypes can arise from distinct KRT5 mutations, even from an identical mutation in one pedigree (13), substitutions in the Glu168 site primarily result in the most severe subtype of EBS, which is EBS-gen sev. However, there exist exceptions (for instance, Glu168Asp results in EBS-gen intermed), suggesting that other factors, such as epigenetic alterations, interchain interactions of protein structure, modifier genes, environmental interference and ethnic background, may exert effects that result in distinct phenotypes.

In the present study, it can be suggested that Glu168Gly is the pathogenic mutation present in the proband based on the following: i) Glu168 is highly conserved among different species (analyzed by the Mutation Taster software; (www.mutationtaster.org/); ii) functional consequence predictions are deleterious; iii) other pathogenic mutations in Glu168 have been reported (Fig. 3); and iv) the variant was not present in the patient's unaffected parents and 100 healthy controls. In combination with the generalized herpetiform blistering occurring since birth and improving with age, the 2-year-old male in the present study was diagnosed with EBS-gen sev.

The patient did not evidently improve after 9 months from the first time that they appeared at the Department of Dermatology of Xinhua Hospital (Shanghai, China), and this may be attributed to the relatively long-term clinical course of EBS-gen sev, or due to insufficient general management of EBS in the infant. Furthermore, no treatment was given within these 5 months. Subsequent general therapy of EBS-gen sev in this patient should concern the prevention of skin trauma, infection control and the maintenance of good nutrition. Since a favorable lifelong prognosis of EBS-gen sev is anticipated following the high mortality rate period (within a year from birth), prenatal diagnosis and potential gene therapy will be available to the next generation in the family.

In conclusion, the current study successfully confirmed a diagnosis of EBS-gen sev by revealing a novel de novo heterozygous missense mutation c. $503 \mathrm{~A}>\mathrm{G}$ in the HIP of KRT5, expanding the existing mutation spectrum.

\section{Acknowledgements}

The authors would like to thank all subjects for their ongoing participation in the study. The present study was supported by grants from the PhD Programs Foundation of the Ministry of Education of China (grant no. 20130073120014) and the Natural Science Foundation of Shanghai Jiaotong University School of Medicine (grant no. 13XJ10023).

\section{References}

1. Fine JD, Bruckner-Tuderman L, Eady RA, Bauer EA, Bauer JW, Has C, Heagerty A, Hintner H, Hovnanian A, Jonkman MF, et al: Inherited epidermolysis bullosa: Updated recommendations on diagnosis and classification. J Am Acad Dermatol 70: 1103-1126, 2014.

2. Coulombe PA, Hutton ME, Vassar R and Fuchs E: A function for keratins and a common thread among different types of epidermolysis bullosa simplex diseases. J Cell Biol 115: 1661-1674, 1991.
3. Coulombe PA, Hutton ME, Letal A, Hebert A, Paller AS and Fuchs E: Point mutations in human keratin 14 genes of epidermolysis bullosa simplex patients: Genetic and functional analyses. Cell 66: 1301-1311, 1991.

4. Hiremagalore R, Kubba A, Bansel S and Jerajani H: Immunofluorescence mapping in inherited epidermolysis bullosa: A study of 86 cases from India. Br J Dermatol 172: 384-391, 2015.

5. Intong LR and Murrell DF: Inherited epidermolysis bullosa: New diagnostic criteria and classification. Clin Dermatol 30: 70-77, 2012.

6. Fine JD, Johnson LB, Weiner M, Li KP and Suchindran C: Epidermolysis bullosa and the risk of life-threatening cancers: The National EB Registry experience, 1986-2006. J Am Acad Dermatol 60: 203-211, 2009.

7. Sawamura D, Nakano H and Matsuzaki Y: Overview of epidermolysis bullosa. J Dermatol 37: 214-219, 2010.

8. Yasukawa K, Sawamura D, Goto M, Nakamura H, Jung SY, Kim SC and Shimizu H: Epidermolysis bullosa simplex in Japanese and Korean patients: Genetic studies in 19 cases. Br J Dermatol 155: 313-317, 2006

9. Steinert PM, Marekov LN, Fraser RD and Parry DA: Keratin intermediate filament structure: Crosslinking studies yield quantitative information on molecular dimensions and mechanism of assembly. J Mol Biol 230: 436-452, 1993.

10. Li XL, Xiao SX, Peng ZH, Liu Y, Pan M and Zhou SN: A mutation in exon 1 of keratin 14 resulting in a Chinese family with epidermolysis bullosa simplex Dowling-Meara. J Eur Acad Dermatol Venereol 21: 979-981, 2007.

11. Yuan H,Liu F, Xiao B,He Y,Liang Y and Liu J: Mutation screening of entire keratin 5 and keratin 14 genes and identification of a novel mutation in a Chinese family with epidermolysis bullosa simplex Dowling-Meara. J Eur Acad Dermatol Venereol 22: $1510-1512,2008$.

12. Wu JW and Xiao SX: A recurrent keratin 14 mutation in Dowling-Meara epidermolysis bullosa simplex in a Chinese family. J Eur Acad Dermatol Venereol 23: 484-486, 2009.

13. Deng W, Yuan P, Lai W, Chen M, Wang Y and Dai S: A novel KRT5 mutation, p.Lys199Asn, is associated with three subtypes of epidermolysis bullosa simplex phenotypes in a single Chinese family. J Dermatol Sci 64: 241-243, 2011.

14. Kang TW, Lee JS, Kim SE, Oh SW and Kim SC: Novel and recurrent mutations in Keratin 5 and 14 in Korean patients with Epidermolysis bullosa simplex. J Dermatol Sci 57: 90-94, 2010.

15. Shinkuma S, Nishie W, Jacyk WK, Natsuga K, Ujiie H, Nakamura H, Akiyama $M$ and Shimizu H: A novel keratin 5 mutation in an african family with epidermolysis bullosa simplex indicates the importance of the amino acid located at the boundary site between the H1 and coil 1A domains. Acta Derm Venereol 93: 585-587, 2013.

16. Müller FB, Küster W, Wodecki K, Almeida $\mathrm{H} \mathrm{Jr}$, Bruckner-Tuderman L, Krieg T, Korge BP and Arin MJ: Novel and recurrent mutations in keratin KRT5 and KRT14 genes in epidermolysis bullosa simplex: Implications for disease phenotype and keratin filament assembly. Hum Mutat 27: 719-720, 2006.

17. Murrell DF, Trisnowati N, Miyakis S and Paller AS: The yin and the yang of keratin amino acid substitutions and epidermolysis bullosa simplex. J Invest Dermatol 131: 1787-1790, 2011.

18. Bolling MC, Lemmink HH, Jansen GH and Jonkman MF: Mutations in KRT5 and KRT14 cause epidermolysis bullosa simplex in 75\% of the patients. Br J Dermatol 164: 637-644, 2011.

19. Stephens K, Ehrlich P, Weaver M, Le R, Spencer A and Sybert VP: Primers for exon-specific amplification of the KRT5 gene: Identification of novel and recurrent mutations in epidermolysis bullosa simplex patients. J Invest Dermatol 108: 349-353, 1997.

20. Betz RC, Planko L, Eigelshoven S, Hanneken S, Pasternack SM, Bussow H, Van Den Bogaert K, Wenzel J, Braun-Falco M, Rutten A, et al: Loss-of-function mutations in the keratin 5 gene lead to Dowling-Degos disease. Am J Hum Genet 78: 510-519, 2006.

21. Guo L, Luo X, Zhao A, Huang H, Wei Z, Chen L, Qin S, Shao L, Xuan J, Feng G, et al: A novel heterozygous nonsense mutation of keratin 5 in a Chinese family with Dowling-Degos disease. J Eur Acad Dermatol Venereol 26: 908-910, 2012.

22. Liao H, Zhao Y, Baty DU, McGrath JA, Mellerio JE and McLean WH: A heterozygous frameshift mutation in the V1 domain of keratin 5 in a family with Dowling-Degos disease. J Invest Dermatol 127: 298-300, 2007.

23. Planko L, Böhse K, Höhfeld J, Betz RC, Hanneken S, Eigelshoven S, Kruse R, Nöthen MM and Magin TM: Identification of a keratin-associated protein with a putative role in vesicle transport. Eur J Cell Biol 86: 827-839, 2007. 